\title{
Alpha Angle as a Predictor of Impending Contralateral Slipped Capital Femoral Epiphysis in an Asian Population
}

\author{
Chloe Xiaoyun Chan, MBBS, Youheng Ou Yang, FRCS, Gloria Hui Min Cheng, MBBS, \\ Sumanth Kumar Gera, MS, Ashik bin Zainuddin Mohammad, FRCSEd \\ Department of Orthopaedic Surgery, KK Women's and Children's Hospital, Singapore
}

Background: Prophylactic pinning of the uninvolved side after unilateral slipped capital femoral epiphysis (SCFE) is controversial. The alpha angle, a measurement of femoral head-neck aspherity, was proposed as a predictor of progression of contralateral SCFE with a treatment threshold of greater than $50.5^{\circ}$. The aim of this study was to evaluate its validity in our cohort of patients.

Methods: A retrospective review of a 10-year series of patients who presented with unilateral SCFE was conducted. Minimum follow-up duration to identify contralateral progression was 18 months. Age, sex, ethnicity, and endocrinopathies were noted. Alpha angle measurements of the unaffected hip were performed by two independent observers. The average values of measurements were used for analysis. Univariate and multivariate logistic regression analyses were performed to identify predictors of contralateral progression. A receiver operating characteristic (ROC) curve was generated.

Results: There were 43 patients with unilateral SCFE. Seven patients (16.3\%) developed contralateral SCFE. There were 31 males $(72.1 \%)$ and 12 females $(27.9 \%)$. The mean duration from index surgery to contralateral fixation was 43.9 weeks (range, 16.2 to 77 weeks). The mean alpha angle was significantly higher in the patients with contralateral progression (mean, 50.7\%; standard

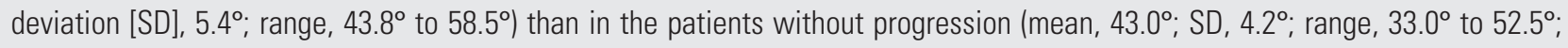
$p<0.001)$. The alpha angle was also identified as a statistically significant predictor of contralateral progression on multivariate analysis $(p=0.02)$. The intraclass correlation coefficient for interobserver reliability was moderately strong at 0.76 ( $95 \%$ confidence interval, 0.55 to 0.87 ). The area under the ROC curve was 0.88 . The treatment threshold of $50.5^{\circ}$ had a sensitivity of 0.43 , specificity of 0.94 , and number needed to treat (NNT) of 2.7. The ideal treatment threshold derived from the ROC curve was $49.0^{\circ}$, which had a sensitivity of 0.71 , specificity of 0.89 , and an NNT of 1.7 .

Conclusions: Alpha angle is a potential predictor of contralateral hip involvement in children with SCFE who may benefit from prophylactic hip fixation. Results from our series suggest a treatment threshold be $49.0^{\circ}$. However, given the limited sample size and moderately strong interobserver reliability, larger studies are needed to validate our findings.

Keywords: Hip, Slipped capital femoral epiphysis, Alpha angle, Bilateral progression

Slipped capital femoral epiphysis (SCFE) is a common hip disorder in adolescents with a reported incidence of 0.2 to

Received March 11, 2019; Accepted June 5, 2019

Correspondence to: Chloe Xiaoyun Chan, MBBS

Department of Orthopaedic Surgery, KK Women's and Children's Hospital, 100 Bukit Timah Rd, Level 4, Children's Tower, Singapore 229899, Singapore

Tel: +65-63942171, Fax: +65-62919232, E-mail: chloe.chan@mohh.com.sg
10 per $100,000{ }^{1,2)}$ Bilateral slips occur in $18 \%-63 \%$ of patients, ${ }^{2,3)}$ approximately $50 \%$ of whom present with bilateral slips simultaneously. The other 50\% develop sequential slips a short duration later. ${ }^{4,5}$ In children with sequential slips, at least $82 \%$ develop a contralateral slip within 18 months of the initial slip. ${ }^{1,3,5)}$

Studies have proposed several SCFE risk factors including ethnicity, ${ }^{6}$ obesity, ${ }^{7)}$ younger age, ${ }^{6}$ modified Oxford bone age score, ${ }^{8)}$ renal insufficiency, ${ }^{9)}$ and endo- 
Chan et al. Alpha Angle as a Predictor of Impending Slipped Capital Femoral Epiphysis

Clinics in Orthopedic Surgery • Vol. 11, No. 4, 2019• www.ecios.org

crine abnormalities such as hypothyroidism and growth hormone deficiency. ${ }^{10)}$ Abnormal mechanical factors, including femoral neck retroversion ${ }^{11)}$ and increased physeal obliquity, ${ }^{12,13)}$ contribute to a weakened physis, resulting in SCFE.

Prophylactic fixation of an unaffected hip has been a highly discussed topic in recent years. Proponents of prophylactic fixation believe that the intervention will prevent deformity and secondary arthritis. ${ }^{13,14)}$ Other surgeons opt for a conservative approach to avoid unnecessary surgery performed on a normal hip. They aim to reduce iatrogenic complications such as pain, loss of motion, development of femoroacetabular impingement (FAI), chondrolysis, avascular necrosis, and peri-implant fractures. ${ }^{15-18)}$ Multiple studies have proposed objective and independent markers that will guide treatment. ${ }^{6-13,19-22)}$ However, the findings have been conflicting and inconclusive.

In 2016, Boyle et al. ${ }^{22}$ described the alpha angle as a significant predictor of an increased likelihood of future contralateral slip, with good interobserver and intraobserver reliability. They proposed a threshold of $50.5^{\circ}$ for prophylactic fixation. The femoral head-neck aspherity, as measured by an elevated alpha angle, ${ }^{23)}$ was thought to potentially contribute to capital physeal instability in patients with SCFE. Since patients with elevated alpha angles are predisposed to cam-type $\mathrm{FAI}^{24)}$ it can be inferred that repetitive femoroacetabular contact would aggravate physeal instability.

In this study, we set out to assess the utility of the alpha angle as a predictor of contralateral slip at our institution with an Asian cohort and to derive an ideal treatment threshold to aid in the management of future patients. Considering morphological differences between Chinese and Caucasian hips, ${ }^{25)}$ the outcome of our study will contribute to our institution's decision analysis model and treatment of populations with similar patient demographics. To our knowledge, this is the first publication reviewing the alpha angle in SCFE in an Asian cohort.

\section{METHODS}

\section{Study Design}

A 10-year (January 2005-April 2015) series of patients who had presented to a large tertiary pediatric public hospital and had been diagnosed as having SCFE were retrospectively reviewed. Appropriate follow-up strategies were used for a minimum of 18 months: serial clinical and radiological evaluations were performed to identify contralateral progression.

Initially, the medical record showed that there are
57 patients with SCFE. Of these, we excluded patients who were lost to follow-up $(n=9)$ and presented with bilateral slips at presentation $(n=5)$. All patients were screened for symptoms or signs of contralateral hip pain, suggestive of possible undiagnosed SCFE, at the first encounter. The remaining 43 patients were divided into two groups: unilateral slip group (unilateral SCFE without clinical or radiological evidence of contralateral hip involvement during follow-up) and contralateral progression group (subsequent progression to contralateral SCFE within 18 months). Clinical evidence of hip involvement was defined as pain, limited weight-bearing, or loss of range of motion. Radiological evidence of SCFE was defined as the presence of an abnormal Klein's sign, the metaphyseal blanch sign of Steel, or frank slippage. Information regarding patient demographics such as age, sex, ethnicity, Loder class, presence of endocrinopathies, renal impairment, radiation exposure, site of injury, and time to contralateral fixation were reviewed.

\section{Measurement of Alpha Angle}

The measurements were performed on the frog-leg lateral radiograph of the unaffected and asymptomatic hip, obtained either just before surgery of the affected hip or in the immediate postoperative period if not obtained preoperatively. To obtain a standardized frog-leg lateral view, patients were laid supine, with the limb of interest flexed approximately $30^{\circ}$ to $40^{\circ}$ and the hip abducted $45^{\circ}$. All radiographs of the unaffected contralateral hip used for alpha angle measurement were taken within one week of surgical pinning of the affected hip and hence would

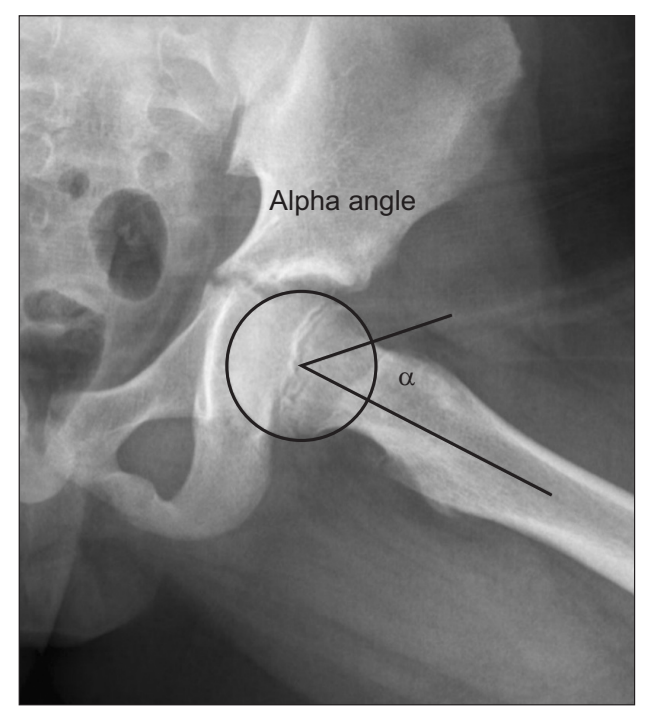

Fig. 1. Measurement of the alpha angle. 
Chan et al. Alpha Angle as a Predictor of Impending Slipped Capital Femoral Epiphysis

Clinics in Orthopedic Surgery • Vol. 11, No. 4, 2019• www.ecios.org

be a true reflection of the state of contralateral hips for the study of prophylactic bilateral pinning in the same setting. The frog-leg lateral radiograph was obtained with patients in supine position, both hips externally rotated and flexed, and the femur abducted as widely as tolerated, forming a frog-leg position. The X-ray beam was directed anteriorly and posteriorly across the joint.

The alpha angle measurements were performed according to the method described by Notzli et al. ${ }^{23)}$ (Fig. 1). First, a best-fit circle was placed over the femoral head.
Two lines were subsequently drawn to form the alpha angle. A first line is drawn from the center of the best-fit circle to the center of the femoral neck at its narrowest point. This line extends along the long axis of the femoral neck. A second line is drawn from the center of the best-fit circle to the point where the femoral neck intersects beyond the boundary of the best-fit circle. The angle formed between these two lines is defined as the alpha angle-an objective measurement of femoral head-neck junction convexity, where a larger angle corresponds to less concavity.

\section{Table 1. Demographic Characteristics and Alpha Angle Values of Patients}

\begin{tabular}{|c|c|c|c|}
\hline Variable & Unilateral slip & Contralateral progression & $p$-value \\
\hline Sex & & & 0.34 \\
\hline Total & 36 & 7 & \\
\hline Male & $27(75.0)$ & $4(57.0)$ & \\
\hline Female & $9(25.0)$ & $3(43.0)$ & \\
\hline
\end{tabular}

Age at diagnosis (yr)

\begin{tabular}{|c|c|c|c|}
\hline Total & $12.2 \pm 1.79(6-15)$ & $12.3 \pm 1.70(9-14)$ & 0.31 \\
\hline Male & $12.6 \pm 1.78(6-15)$ & $12.6 \pm 0.96(12-14)$ & 0.40 \\
\hline Female & $10.8 \pm 0.83(10-12)$ & $11.7 \pm 2.52(9-14)$ & 1.0 \\
\hline \multicolumn{4}{|l|}{ Ethnicity } \\
\hline Chinese & $10(27.8)$ & $3(42.9)$ & 0.43 \\
\hline Indian & $16(44.4)$ & $2(28.6)$ & 0.44 \\
\hline Malay & $7(19.4)$ & $2(28.6)$ & 0.59 \\
\hline Others & $3(8.3)$ & 0 & - \\
\hline Site of initial slip & & & 0.14 \\
\hline Left & $16(44.0)$ & $1(14.2)$ & \\
\hline Right & $20(56.0)$ & $6(85.7)$ & \\
\hline
\end{tabular}

Loder class

\begin{tabular}{|rrr}
\hline Stable & $27(75.0)$ & $5(71.4)$ \\
\hline Unstable & $9(25.0)$ & $2(28.6)$
\end{tabular}

Medical comorbidity

Endocrinopathy

6 (Impaired fasting glucose)

1 (Type 2 diabetes mellitus)

0.88

Renal impairment

0

0

Radiation exposure

0

0

Time to contralateral fixation (wk)

NA

$43.7(16.2-77)$

Alpha angle $\left(^{\circ}\right) \quad 43.0 \pm 4.2(33.0-52.5)$

$50.7 \pm 5.4(43.8-58.5)$

$<0.001$

Values are presented as number (\%) or mean \pm standard deviation (range).

NA: not applicable. 
Chan et al. Alpha Angle as a Predictor of Impending Slipped Capital Femoral Epiphysis

Clinics in Orthopedic Surgery • Vol. 11, No. 4, 2019• www.ecios.org

The measurements were conducted by Chloe Chan, Gloria Cheng who are part of the study team (GHMC, $\mathrm{CXC}$ ), in an independent and a blinded fashion and assessed for interobserver reliability via the intraclass correlation coefficient (ICC). The average values of measurements obtained by the two trainees were used for analysis.

\section{Statistical Analysis}

Categorical variables are presented as frequency and percentage and analyzed across groups (unilateral slip group and contralateral progression group) by using the chisquare test. Continuous variables are presented as mean \pm standard deviation (SD) and range. Comparisons were made by using the unpaired, two-tailed $t$-test for characteristics that meet the assumptions of normality; and the Mann-Whitney $U$-test for characteristics that deviate from normality. The null hypothesis was that there would be no significant difference in the alpha angle between groups. Multivariate logistic regression analysis was performed to identify independent factors for contralateral progression while adjusting for possible confounders. Level of significance was set to be below 0.05 . A receiver operating characteristic (ROC) curve was generated with the average alpha angle measurements to determine the best treatment threshold. All data were compiled in Microsoft Excel 2003 (Microsoft Corp., Redmond, WA, USA) and the statisti- cal analysis was performed with IBM SPSS ver. 23.0 (IBM Corp., Armonk, NY, USA).

\section{Ethical Aspect}

This study was ethically approved by a Centralized Institutional Review Board of KK Children's and Women's Hospital with a waiver of consent (reference No. 2016/3143).

\section{RESULTS}

A total of 43 patients were included in the analysis. During the 18-month follow-up, 36 patients (83.7\%) remained stable with a unilateral slip whilst seven patients (16.3\%) had contralateral progression. The overall sex distribution of the study population was 31 males $(72.1 \%)$ and 12 females (27.9\%). The average age at the time of diagnosis was 12.2 years (range, 6 to 15 years): 12.6 (range, 6 to 15 years) in males and 10.8 years (range, 10 to 12 years) in females. The ethnic distribution was as follows: 13 (30.2\%), Chinese; nine (20.9\%), Malay; 18 (41.8\%), Indian; and three $(7.0 \%)$, others.

Amongst the 43 slips diagnosed at presentation, 17 (39.5\%) occurred in the left hip and 26 (60.5\%) occurred in the right hip. The Loder class was found to be unstable in 11 hips (25.6\%) and stable in 32 hips (74.4\%). Endocrinopathies were found in seven patients: impaired fasting

Table 2. Multivariate Logistic Regression Analysis for Association between Alpha Angle and Other Independent Variables and Contralateral Progression ( $\mathrm{N}=43)$

\begin{tabular}{|c|c|c|c|c|}
\hline Variable & Unilateral slip & Contralateral progression & $p$-value & Odds ratio $(95 \% \mathrm{CI})$ \\
\hline Sex & & & 0.711 & $2.40(0.02-248.58)$ \\
\hline Male & $27(75.0)$ & $4(57.0)$ & & \\
\hline Female & $9(25.0)$ & $3(43.0)$ & & \\
\hline Ethnicity & & & & - \\
\hline Chinese & $10(27.8)$ & $3(42.9)$ & 0.58 & \\
\hline Indian & $16(44.4)$ & $2(28.6)$ & 1.00 & \\
\hline Malay & $7(19.4)$ & $2(28.6)$ & 1.00 & \\
\hline Others & $3(8.3)$ & 0 & 1.00 & \\
\hline Medical comorbidity & & & & $12.6(0-752,897.23)$ \\
\hline Endocrinopathy & 6 (Impaired fasting glucose) & 1 (Type 2 diabetes mellitus ) & 0.65 & \\
\hline Renal impairment & 0 & 0 & - & \\
\hline Radiation exposure & 0 & 0 & - & - \\
\hline Alpha angle $\left({ }^{\circ}\right)$ & $43.0 \pm 4.2(33.0-52.5)$ & $50.7 \pm 5.4(43.8-58.5)$ & 0.02 & $2.19(1.13-4.26)$ \\
\hline
\end{tabular}

Values are presented as number (\%) or mean \pm standard deviation (range). 
Chan et al. Alpha Angle as a Predictor of Impending Slipped Capital Femoral Epiphysis

Clinics in Orthopedic Surgery • Vol. 11, No. 4, 2019• www.ecios.org

glucose in six patients in the unilateral slip group and type 2 diabetes in one patient in the contralateral progression group. Renal impairment and prior radiation exposure were absent in all patients. There were no statistical differences between groups in the sex, age of onset, site of slip, Loder class, and presence of medical comorbidities $(p>$ $0.05)$. The mean interval from index surgery to contralateral fixation was 43.9 weeks (range, 16.2 to 77 weeks).

\section{Alpha Angle}

The mean alpha angle was higher in the contralateral progression group with $50.7^{\circ}$ (SD, $5.4^{\circ}$; range, $43.8^{\circ}$ to $58.5^{\circ}$ ) compared to the unilateral group with $43.0^{\circ}$ (SD, $4.2^{\circ}$; range, $33.0^{\circ}$ to $52.5^{\circ}$ ) (Table 1 ). The difference was statistically significant $(p<0.001)$. The alpha angle was also identified as a statistically significant predictor of contralateral progression on multivariate analysis $(p=0.02)$ (Table 2 ). The ICC was moderately strong at 0.76 (95\% confidence

Table 3. Comparison of Data with Those of Boyle et al ${ }^{221}$ on Alpha Angle and Slipped Capital Femoral Epiphysis

\begin{tabular}{|c|c|c|}
\hline Variable & This study & Boyle et al. $^{22)}$ \\
\hline Year of publication & 2019 & 2016 \\
\hline Study setting & Singapore & USA \\
\hline Total no. of patients & 43 & 168 \\
\hline Male sex (\%) & 72.1 & 56.0 \\
\hline \multicolumn{3}{|l|}{ Age at diagnosis (yr) } \\
\hline Unilateral & $12.2 \pm 1.79$ & $12.2 \pm 1.52$ \\
\hline Contralateral progression & $12.3 \pm 1.70$ & $12.2 \pm 1.50$ \\
\hline \multicolumn{3}{|l|}{ No. of patients } \\
\hline Unilateral & 36 & 123 \\
\hline Contralateral progression & $7(16.3)$ & 45 (26.8) \\
\hline \multicolumn{3}{|c|}{ Average posterior sloping angle $\left(^{\circ}\right)$} \\
\hline Unilateral & $43.0 \pm 4.2$ & $44.9 \pm 5.3$ \\
\hline Contralateral progression & $50.7 \pm 5.4$ & $50.6 \pm 8.8$ \\
\hline Treatment threshold $\left({ }^{\circ}\right)$ & 49.0 & 50.5 \\
\hline Area under the ROC curve & 0.88 & 0.68 \\
\hline Number needed to treat & 1.7 & 2.7 \\
\hline Predicted sensitivity & 0.71 & 0.58 \\
\hline Predicted specificity & 0.89 & 0.85 \\
\hline
\end{tabular}

Values are presented as mean \pm standard deviation or number (\%). ROC curve: receiver operating characteristic curve. interval [CI], 0.55 to 0.87 ) between observers. The area under the ROC curve was 0.88 - a greater area under the curve closer to the value of 1 supports the use of alpha angle in predicting the likelihood of sustaining contralateral SCFE. The ROC-derived ideal treatment threshold of $49.0^{\circ}$ had a sensitivity 0.71 , specificity of 0.89 , and number needed to treat (NNT) of 1.7 (Fig. 2). In contrast, a treatment threshold of $50.5^{\circ}$ suggested by Boyle et al. ${ }^{22)}$ had a lower sensitivity of 0.43 , a specificity of 0.94 , and an NNT of 2.7 .

\section{DISCUSSION}

The discussion regarding prophylactic fixation of a contralateral, asymptomatic, normal hip in patients with SCFE arose in view of the high rates of bilateral or sequential slips. The prophylactic approach confers a theoretical advantage of reducing the risk of osteoarthritis and deformity, particularly in delayed diagnosis where there is a higher possibility of slips being moderate to severe in nature. ${ }^{26,27)}$

To date, there is an increasing focus on objective predictors of contralateral involvement such as the modified Oxford bone age score and the posterior sloping angle (easily obtained, reliable radiological measurement), in addition to traditional predictors like younger age and presence of endocrinological or renal abnormalities. In 2016, Boyle et al. ${ }^{22)}$ proposed the alpha angle as an additional tool in the decision-making process. While current recommendations still suggest a multipronged approach in risk stratification, ${ }^{2,13,14,17)}$ our aim was to evaluate the validity of the alpha angle in our SCFE population.

The alpha angle is a measurement of femoral headneck aspherity. It was originally used to determine anterior

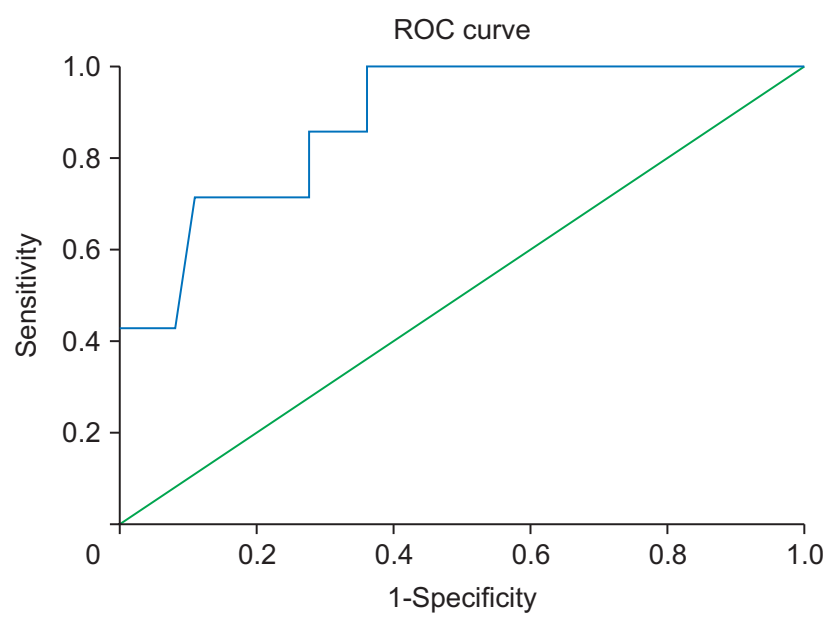

Fig. 2. The receiver operating characteristic (ROC) curve for alpha angle with respect to contralateral progression. 
Chan et al. Alpha Angle as a Predictor of Impending Slipped Capital Femoral Epiphysis

Clinics in Orthopedic Surgery • Vol. 11, No. 4, $2019 \bullet$ www.ecios.org

impingement of the femoral neck on the acetabular rim or labrum, in the context of FAI syndrome. It has been proposed that the pistol grip deformity in patients with FAI is a result of previously undiagnosed developmental deformity. ${ }^{23)}$ This association has been reported in subacute cases of SCFE ${ }^{28)}$ Similar to the results of the study of Boyle et al., ${ }^{22)}$ our results suggest a positive relationship between greater femoral head-neck aspherity and risk of developing contralateral SCFE.

Two theories have been proposed by Boyle et al. ${ }^{22)}$ The first theory is a possible increase in transphyseal mechanical stress on the femoral head-neck junction in patients with increased aspherity. This occurs as a result of repetitive subclinical contact with the acetabulum in patients with increased alpha angle, eventually resulting in a SCFE. This theory was similarly proposed by Gelberman et al. ${ }^{11)}$ in patients with femoral neck retroversion. However, increased acetabular depth has no proven significance.

There is also possibility that patients with increased alpha angle have silent SCFE, which subsequently manifests as overt or symptomatic SCFE. As per Lehmann et al., ${ }^{29)} 6.6 \%$ of a cohort of 2,072 healthy adolescents $(58 \%$ females) had radiological findings (Southwick's lateral head-shaft angle $\geq 13^{\circ}$ ) consistent with a prior SCFE. Jerre et al. ${ }^{19)}$ described significant rates of asymptomatic contralateral SCFE of up to $71 \%$ (42/59) on follow-up of patients up to adulthood. While both theories have not been proven, our study adds additional information to existing evidence that femoral head-neck aspherity correlates with symptomatic contralateral SCFE development. The alpha angle is a useful additional tool when considering prophylactic fixation.

Comparing our results with those of Boyle et al. ${ }^{22)}$ (Table 3), despite similar population characteristics, we had a smaller proportion of contralateral progression. Our proposed treatment threshold of $49.0^{\circ}$ gave a higher sensitivity and specificity, with an improved NNT. However, Monazzam et al. ${ }^{30)}$ reported a mean alpha angle of $49.1^{\circ}$ in males and $44.7^{\circ}$ in females aged 12 to 13 years. There is possibility that a treatment threshold of $49.0^{\circ}$ may be too narrow, especially in the context of other ethnicities. A study of the nature of alpha angles with computed tomography in various ethnicities by Gollwitzer et al. ${ }^{31)}$ suggests that Caucasians and Africans have a significantly higher alpha angle than Asians. This may account for the differ- ence in treatment thresholds between our cohort and that of Boyle et al. ${ }^{22)}$ Ultimately, our result is supportive of the utility of alpha angle as a predictor of impending contralateral SCFE.

To the best of our knowledge, this is the first study on the alpha angle in SCFE in the Asian population, and the second published study to address alpha angle in SCFE. The strength of our paper lies in the minimum 18 months of clinical and radiological follow-up in a cohort of 10 consecutive years. Two independent researchers conducted measurements of the alpha angle with a moderate degree of reliability (ICC, 0.76 ). While the value was lower than the ICC of 0.92 reported by Boyle et al., ${ }^{22)}$ it adds credibility to the objectiveness of the alpha angle.

Limitations to this study include the retrospective nature of data collection and results that were generated from a single center. In addition, the sample size was small despite the study includes a 10-year series. However, based on the previous demographic study performed in Singapore, ${ }^{5)}$ the rate of SCFE in our population is comparatively low. As body mass index data were not available, we were unable to analyze the effect of obesity on the progression to contralateral slip, although other risk factors such as atypical SCFE, age of onset, gender and endocrinopathies were accounted for.

In conclusion, an increased alpha angle was significantly associated with contralateral hip involvement in children with SCFE. A treatment threshold of $49.0^{\circ}$ yielded a $71 \%$ sensitivity and $89 \%$ specificity for contralateral slippage with an NNT of only 1.7. However, given our limited sample size and the moderately strong interobserver reliability, larger studies are needed to validate our findings. As prophylactic treatment is a major intervention with risks of iatrogenic complications, the management of patients should not be guided by an isolated value alone. The alpha angle need to be combined with other measurements to form a multifactorial scoring system that may provide an optimal treatment threshold for prophylactic fixation.

\section{CONFLICT OF INTEREST}

No potential conflict of interest relevant to this article was reported.

\section{REFERENCES}

1. Loder RT. The demographics of slipped capital femoral

epiphysis: an international multicenter study. Clin Orthop 
Chan et al. Alpha Angle as a Predictor of Impending Slipped Capital Femoral Epiphysis

Clinics in Orthopedic Surgery • Vol. 11, No. 4, 2019• www.ecios.org

Relat Res. 1996;(322):8-27.

2. Aronsson DD, Loder RT, Breur GJ, Weinstein SL. Slipped capital femoral epiphysis: current concepts. J Am Acad Orthop Surg. 2006;14(12):666-79.

3. Castro FP Jr, Bennett JT, Doulens K. Epidemiological perspective on prophylactic pinning in patients with unilateral slipped capital femoral epiphysis. J Pediatr Orthop. 2000;20(6):745-8.

4. Loder RT, Aronson DD, Greenfield ML. The epidemiology of bilateral slipped capital femoral epiphysis: a study of children in Michigan. J Bone Joint Surg Am. 1993;75(8):1141-7.

5. Lim YJ, Lam KS, Lee EH. Review of the management outcome of slipped capital femoral epiphysis and the role of prophylactic contra-lateral pinning re-examined. Ann Acad Med Singapore. 2008;37(3):184-7.

6. Bidwell TA, Susan Stott N. Sequential slipped capital femoral epiphyses: who is at risk for a second slip? ANZ J Surg. 2006;76(11):973-6.

7. Nasreddine AY, Heyworth BE, Zurakowski D, Kocher MS. A reduction in body mass index lowers risk for bilateral slipped capital femoral epiphysis. Clin Orthop Relat Res. 2013;471(7):2137-44.

8. Popejoy D, Emara K, Birch J. Prediction of contralateral slipped capital femoral epiphysis using the modified Oxford bone age score. J Pediatr Orthop. 2012;32(3):290-4.

9. Loder RT, Hensinger RN. Slipped capital femoral epiphysis associated with renal failure osteodystrophy. J Pediatr Orthop. 1997;17(2):205-11.

10. Loder RT, Wittenberg B, DeSilva G. Slipped capital femoral epiphysis associated with endocrine disorders. J Pediatr Orthop. 1995;15(3):349-56.

11. Gelberman RH, Cohen MS, Shaw BA, Kasser JR, Griffin PP, Wilkinson RH. The association of femoral retroversion with slipped capital femoral epiphysis. J Bone Joint Surg Am. 1986;68(7):1000-7.

12. Mirkopulos N, Weiner DS, Askew M. The evolving slope of the proximal femoral growth plate relationship to slipped capital femoral epiphysis. J Pediatr Orthop. 1988;8(3):26873.

13. Phillips PM, Phadnis J, Willoughby R, Hunt L. Posterior sloping angle as a predictor of contralateral slip in slipped capital femoral epiphysis. J Bone Joint Surg Am. 2013;95(2):146-50.

14. Hagglund G. Pinning the slipped and contralateral hips in the treatment of slipped capital femoral epiphysis. J Child Orthop. 2017;11(2):110-3.

15. Sankar WN, Novais EN, Lee C, Al-Omari AA, Choi PD,
Shore BJ. What are the risks of prophylactic pinning to prevent contralateral slipped capital femoral epiphysis? Clin Orthop Relat Res. 2013;471(7):2118-23.

16. Kumm DA, Schmidt J, Eisenburger SH, Rutt J, Hackenbroch $\mathrm{MH}$. Prophylactic dynamic screw fixation of the asymptomatic hip in slipped capital femoral epiphysis. J Pediatr Orthop. 1996;16(2):249-53.

17. Schultz WR, Weinstein JN, Weinstein SL, Smith BG. Prophylactic pinning of the contralateral hip in slipped capital femoral epiphysis: evaluation of long-term outcome for the contralateral hip with use of decision analysis. J Bone Joint Surg Am. 2002;84(8):1305-14.

18. Yildirim Y, Bautista S, Davidson RS. Chondrolysis, osteonecrosis, and slip severity in patients with subsequent contralateral slipped capital femoral epiphysis. J Bone Joint Surg Am. 2008;90(3):485-92.

19. Jerre R, Billing L, Hansson G, Karlsson J, Wallin J. Bilaterality in slipped capital femoral epiphysis: importance of a reliable radiographic method. J Pediatr Orthop B. 1996;5(2):804.

20. Park S, Hsu JE, Rendon N, Wolfgruber H, Wells L. The utility of posterior sloping angle in predicting contralateral slipped capital femoral epiphysis. J Pediatr Orthop. 2010;30(7):683-9.

21. Mestriner MB, Verquietini CM, Waisberg G, Akkari M, Fukunaga ET, Santili C. Radiographic evaluation in epiphysiolysis: possible predictors of bilaterality? Acta Ortop Bras. 2012;20(4):203-6.

22. Boyle MJ, Lirola JF, Hogue GD, Yen YM, Millis MB, Kim YJ. The alpha angle as a predictor of contralateral slipped capital femoral epiphysis. J Child Orthop. 2016;10(3):201-7.

23. Notzli HP, Wyss TF, Stoecklin CH, Schmid MR, Treiber K, Hodler J. The contour of the femoral head-neck junction as a predictor for the risk of anterior impingement. J Bone Joint Surg Br. 2002;84(4):556-60.

24. Beck M, Kalhor M, Leunig M, Ganz R. Hip morphology influences the pattern of damage to the acetabular cartilage: femoroacetabular impingement as a cause of early osteoarthritis of the hip. J Bone Joint Surg Br. 2005;87(7):1012-8.

25. Dudda M, Kim YJ, Zhang Y, et al. Morphologic differences between the hips of Chinese women and white women: could they account for the ethnic difference in the prevalence of hip osteoarthritis? Arthritis Rheum. 2011;63(10):2992-9.

26. Erickson JB, Samora WP, Klingele KE. Treatment of chronic, stable slipped capital femoral epiphysis via surgical hip dislocation with combined osteochondroplasty and Imhauser osteotomy. J Child Orthop. 2017;11(4):284-8. 
Chan et al. Alpha Angle as a Predictor of Impending Slipped Capital Femoral Epiphysis

Clinics in Orthopedic Surgery • Vol. 11, No. 4, 2019• www.ecios.org

27. Sikora-Klak J, Bomar JD, Paik CN, Wenger DR, Upasani $\mathrm{V}$. Comparison of surgical outcomes between a triplane proximal femoral osteotomy and the modified Dunn procedure for stable, moderate to severe slipped capital femoral epiphysis. J Pediatr Orthop. 2019;39(7):339-46.

28. Leunig M, Casillas MM, Hamlet M, et al. Slipped capital femoral epiphysis: early mechanical damage to the acetabular cartilage by a prominent femoral metaphysis. Acta Orthop Scand. 2000;71(4):370-5.

29. Lehmann TG, Engesaeter IO, Laborie LB, Lie SA, Rosendahl $\mathrm{K}$, Engesaeter LB. Radiological findings that may indicate a prior silent slipped capital femoral epiphysis in a cohort of 2072 young adults. Bone Joint J. 2013;95(4):452-8.

30. Monazzam S, Bomar JD, Dwek JR, Hosalkar HS, Pennock AT. Development and prevalence of femoroacetabular impingement-associated morphology in a paediatric and adolescent population: a CT study of 225 patients. Bone Joint J. 2013;95(5):598-604.

31. Gollwitzer H, Suren C, Struwind C, et al. The natural alpha angle of the femoral head-neck junction: a cross-sectional CT study in 1312 femurs. Bone Joint J. 2018;100(5):570-8. 